\title{
Efficient synthesis of $N$-acylbenzotriazoles using tosyl chloride: en route to suberoylanilide hydroxamic acid (SAHA)
}

\author{
Khalid A. Agha, ${ }^{a}$ Nader E. Abo-Dya, ${ }^{\text {a,b }}$ Tarek S. Ibrahim, ${ }^{\text {a }}$ and Eatedal H. Abdel-Aal ${ }^{\text {a }}$ \\ ${ }^{a}$ Department of Pharmaceutical Organic Chemistry, Faculty of Pharmacy, Zagazig University, \\ Zagazig, 44519, Egypt \\ ${ }^{b}$ Department of Pharmaceutical Chemistry, Faculty of Pharmacy, Tabuk University, Tabuk, \\ 71491, Saudi Arabia \\ E-mail: nader_elmaghry88@yahoo.com
}

This paper is dedicated to (the late) Professor Alan R. Katritzky

DOI: http://dx.doi.org/10.3998/ark.5550190.p009.459

\begin{abstract}
Various carboxylic acids were converted into $N$-acylbenzotriazoles (90-97\% isolated yields) via a one-pot synthesis involving activation of carboxylic acids with tosyl chloride. The novel protocol enabled stepwise manipulation of both carboxylic groups of suberic acid en route to Vorinostate (SAHA). In addition to the high yield of SAHA (84\% yield over four steps) the new method comprises a simple work up and short reaction times.
\end{abstract}

Keywords: $N$-Acylbenzotriazole, tosyl chloride, anilides, hydroxamic acids, Vorinostate

\section{Introduction}

Benzotriazole has been used extensively as a versatile synthetic auxiliary. ${ }^{1} \mathrm{~N}$-Acylbenzotriazoles are advantageous acylating agents showing numerous advantages over acid chlorides such as: i) they are isolated in high yields ii) they are usually crystalline iii) they are stable in air and iv) chirality is preserved during the course of their preparation and reaction. $N$-Acylbenzotriazoles are widely used when the corresponding acid chlorides are unstable or difficult to prepare. ${ }^{2}$

$N$ - $\alpha$-Aminoacylbenzotriazoles were synthesized from proteinogenic amino acids and used efficiently as versatile building blocks for construction of peptides, peptide conjugates, peptidomimetics, cyclic peptides and cyclic peptidomimetics. Moreover, benzotriazole methodology enabled the synthesis of fluorescent and dye-labeled peptides as well as the total synthesis of natural cyclic peptide Rolloamide B..$^{3-8}$

Katritzky and co-workers established two methods for the synthesis $N$-acylbenzotriazoles directly from carboxylic acids utilizing thionyl chloride and 1-(methanesulfonyl) benzotriazole 
$\left(\mathrm{BtO}_{2} \mathrm{SMe}\right) .^{5}$ Although the thionyl chloride method is widely applicable, it is not suitable for acid sensitive starting material. ${ }^{5}$

Recently, $\mathrm{N}$-acylbenzotriazoles were synthesized via reacting carboxylic acids with benzotriazole in the presence of TEA using either $\mathrm{Ph}_{3} \mathrm{P} / \mathrm{I}_{2}{ }^{9}$ or $2,4,6$-trichloro-1,3,5-triazine. ${ }^{10}$ These methods are so far limited to simple aliphatic and aromatic carboxylic acids and the $\mathrm{Ph}_{3} \mathrm{P} / \mathrm{I}_{2}$ method requires tedious chromatographic purification of the target compounds.

Herein, carboxylic acids are activated for coupling with benzotriazole using $p$-toluenesulfonyl chloride. This novel method enables benzotriazole-mediated synthesis of SAHA starting from a cheap starting material (suberic acid) in high overall yield (84\%).

\section{Results and Discussion}

$p$-Toluenesulfonyl chloride has been formerly used for activation of carboxylic acids to couple with esters of $\alpha$-amino acids and alcohols. ${ }^{11,12}$ The intermediates of such coupling reaction are thought to be sulfonic carboxylic mixed anhydrides.

In the current work a carboxylic acid is activated to couple with benzotriazole using $p$ toluenesulfonyl chloride. Firstly, $p$-toluenesulfonyl chloride reacts with carboxylic acid in the presence of triethylamine and catalytic amount of DMAP $(0.13$ mole $\%)$ to form mixed sulfonic carboxylic anhydrides $\mathbf{2 a - n}$. Subsequent addition of $1 \mathrm{H}$-benzotriazole furnished $\mathrm{N}$-acylbenzotriazoles 3a-n in $90-96 \%$ isolated yields. The overall reaction time is two hours due to the presence of DMAP which accelerates both the sulfonylation and the acylation steps of the reaction (Scheme 1). ${ }^{13}$

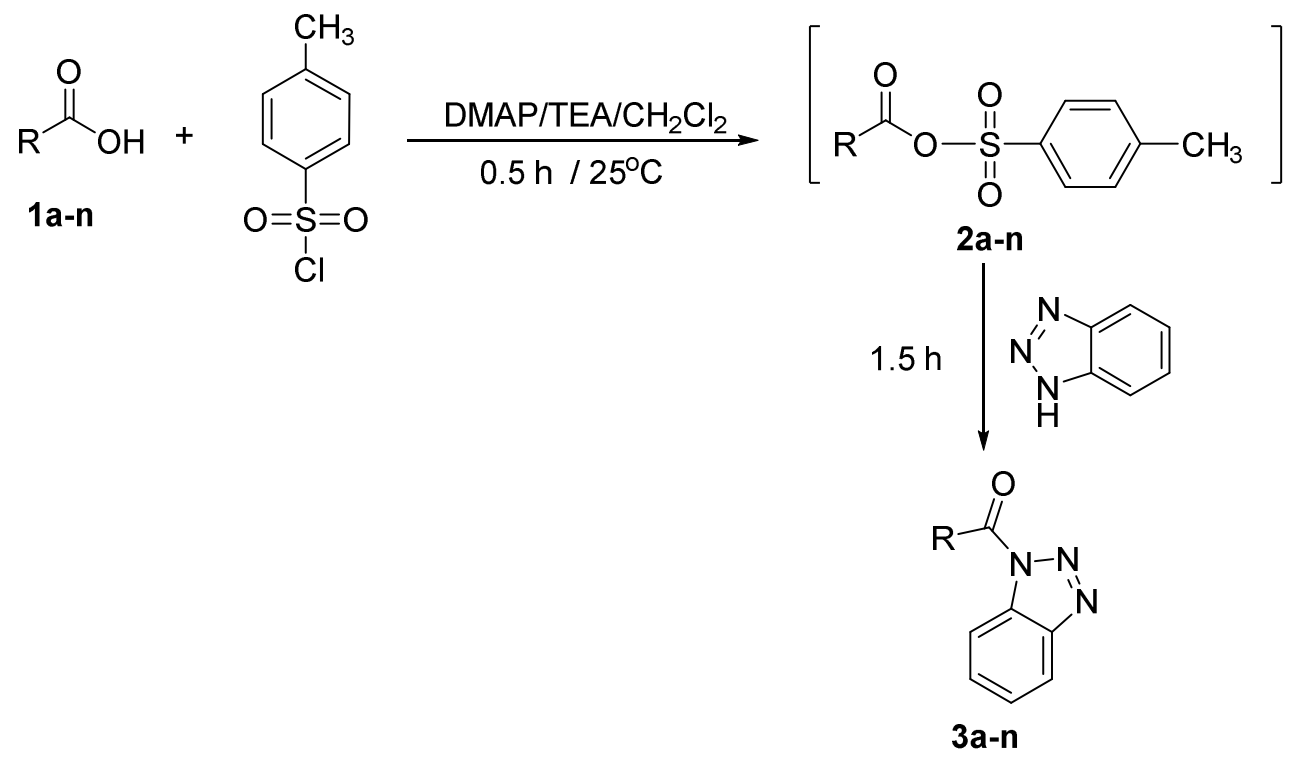

Scheme 1. Synthesis of $N$-acylbenzotriazoles via tosyl activation of carboxylic acids. 
Benzotriazolides were prepared from aliphatic carboxylic acids (1a-b), namely acetic and stearic acids, $N$-L-Tos-Trp-OH (3c) and aromatic carboxylic acids (1d-l) in excellent yields (9096\%). Interestingly, $N$-(3-aminobenzoyl)benzotriazole (3k) and $N$-(4-aminobenzoyl)benzotriazole (3l) were prepared directly from 3-aminobenzoic acid (1k) and 4-aminobenzoic acid (11) in $92-95 \%$ yields without protection of the free amino group. Furthermore, $N$-acylbenzotriazoles (3m-n) were prepared from heterocyclic carboxylic acids (1m-n) in 93-94\% yields. The target compounds were isolated by simple work up and characterized using ${ }^{1} \mathrm{H}$ NMR, ${ }^{13} \mathrm{C}$ NMR and elemental analyses.

Vorinostate (SAHA) is a histone deacetylase inhibitor (HDACI) used as a potent differentiating agent toward breast and prostate cancers. Reported methods for the synthesis of SAHA utilized suberoyl chloride, ${ }^{14}$ suberic acid, ${ }^{15}$ and suberic acid monomethyl ester ${ }^{16}$ as starting materials. The disadvantages of these methods include low overall yields (15-51\%), ${ }^{14-16}$ tedious chomatographic work-up, ${ }^{14}$ and the use of relatively expensive starting materials and reagents. $^{16}$

In the present study reaction of suberic acid (4) with equimolar amount of $p$-toluenesulfonyl chloride and benzotriazole in the presence of TEA gave compound $\mathbf{5}$ in $95 \%$ yield. Stirring of $\mathbf{5}$ with aniline at $25{ }^{\circ} \mathrm{C}$ in methylene chloride for $1 \mathrm{~h}$ affords suberanilic acid 6 in $96 \%$ yield. The carboxylic acid group of $\mathbf{6}$ was converted to the corresponding benzotriazolide using the tosyl activation reported here to produce $N$-acylbenzotriazole 7 in $97 \%$ yield. Reaction of 7 with a mixture of hydroxylamine $\mathrm{HCl}$ and TEA at $25{ }^{\circ} \mathrm{C}$ for $1 \mathrm{~h}$. gave SAHA in $95 \%$ yield. Thus, suberic acid was converted into SAHA in $84 \%$ overall yield (Scheme 2). The advantages of this method are: (i) short reaction times, the four reactions take $5.5 \mathrm{~h}$; (ii) simple work up; (iii) cheap starting materials and reagents; (iv) benzotriazole can be recycled.

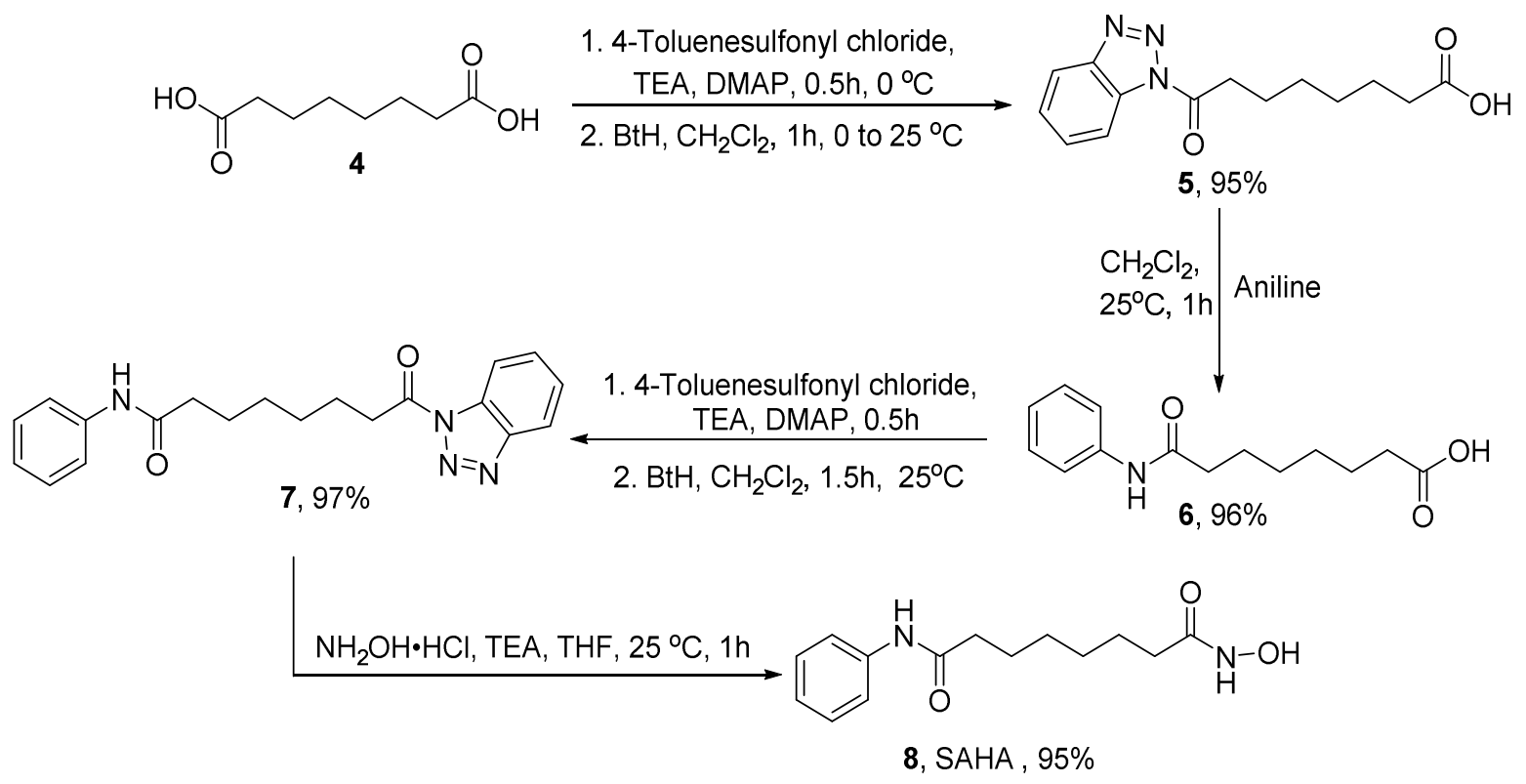

Scheme 2. Benzotriazole-mediated synthesis of SAHA. 


\section{Conclusions}

In conclusion, we have developed a novel and efficient protocol for the synthesis of $N$ acylbenzotriazoles using tosyl chloride for carboxyl activation. The novel procedures enabled the synthesis of SAHA from cheap starting materials in a high overall yield (84\%) and simple work up. In addition, protecting-group-free conversion of 3-aminobenzoic acid and 4-aminobenzoic acid into their corresponding benzotriazolides was accomplished using the tosyl activation protocol described herein.

\section{Experimental Section}

General. Starting materials and solvents were purchased from common commercial sources and used without further purification. Melting points were determined on Fisher melting point apparatus. ${ }^{1} \mathrm{H}$ NMR (400 MHz) and ${ }^{13} \mathrm{C}$ NMR (100 MHz) spectra were recorded on a Bruker 400 MHz NMR spectrometer using DMSO- $d_{6}$ as solvent. The chemical shift $(\delta)$ is reported in ppm, and coupling constants $(J)$ are given in Hz. Elemental analyses were performed on a Carlo Erba1106 instrument. All reactions were monitored by (TLC) with visualization by UV irradiation.

Synthesis of compounds 3a-n. General procedure. A mixture of $p$-toluenesulfonyl chloride $(0.19 \mathrm{~g}, 1 \mathrm{mmol})$ and DMAP $(0.016 \mathrm{~g}, 0.13 \mathrm{mmol})$ was stirred in $\mathrm{CH}_{2} \mathrm{Cl}_{2}(5 \mathrm{~mL})$ for 10 minutes. The carboxylic acid $(1 \mathrm{mmol})$ was dissolved in $\mathrm{CH}_{2} \mathrm{Cl}_{2}(5 \mathrm{~mL})$ containing TEA $(0.21 \mathrm{~mL}, 1.5$ mmol) and the resulting solution was added to the reaction mixture. After 20 minutes, benzotriazole $(0.143 \mathrm{~g}, 1.2 \mathrm{mmol})$ was added and the reaction was allowed to stir for additional $1.5 \mathrm{~h}$ at $25{ }^{\circ} \mathrm{C}$. Upon completion of the reaction (monitored by TLC) $\mathrm{CH}_{2} \mathrm{Cl}_{2}(50 \mathrm{~mL}$ ) was added and the organic layer was washed with saturated $\mathrm{Na}_{2} \mathrm{CO}_{3}(10 \mathrm{~mL}, 3 \mathrm{x})$, water $(10 \mathrm{~mL}, 2 \mathrm{x})$ and brine $(10 \mathrm{~mL}, 1 \mathrm{x})$. The organic layer was dried over anhydrous sodium sulfate and hexane (20 $\mathrm{mL}$ ) was added. The solid separated was filtered and dried under vacuum to give the target $N$ acylbenzotriazoles 3a-n.

1-(1H-1,2,3-Benzotriazol-1-yl)ethanone (3a). White microcrystals, yield $0.148 \mathrm{~g}$ (92\%); mp 49-51 ${ }^{\circ} \mathrm{C}$. (lit. 49-51 $\left.{ }^{\circ} \mathrm{C}\right) .{ }^{17}{ }^{1} \mathrm{H}$ NMR (400 MHz, DMSO- $\left.d_{6}\right) \delta 8.22(\mathrm{t}, J 8.0 \mathrm{~Hz}, 2 \mathrm{H}, \mathrm{Ar}-\mathrm{H}), 7.78$ - $7.74(\mathrm{~m}, 1 \mathrm{H}, \mathrm{Ar}-\mathrm{H}), 7.61-7.57(\mathrm{~m}, 1 \mathrm{H}, \mathrm{Ar}-\mathrm{H}), 2.94\left(\mathrm{~s}, 3 \mathrm{H},-\mathrm{CH}_{3}\right) .{ }^{13} \mathrm{C} \mathrm{NMR}(100 \mathrm{MHz}$, DMSO- $\left.d_{6}\right) \delta 169.6(\mathrm{C}=\mathrm{O}), 145.4(\mathrm{C}-\mathrm{N}=\mathrm{N}), 130.5(\mathrm{C}-\mathrm{N}), 130.4(\mathrm{Ar}-\mathrm{C}), 126.1(\mathrm{Ar}-\mathrm{C}), 119.8(\mathrm{Ar}-$ C), 113.8 (Ar-C), $23.0\left(\mathrm{CH}_{3}\right)$.

1-(1H-1,2,3-Benzotriazol-1-yl)octadecan-1-one (3b). White microcrystals, yield $0.36 \mathrm{~g}(94 \%)$; mp 58-60 ${ }^{\circ} \mathrm{C} .{ }^{1} \mathrm{H}$ NMR (400 MHz, DMSO-d $)_{6} \delta 8.24(\mathrm{~d}, J 8.4 \mathrm{~Hz}, 2 \mathrm{H}, \mathrm{Ar}-\mathrm{H}), 7.77(\mathrm{t}, J 8 \mathrm{~Hz}$, 1H, Ar-H), 7.60 (t, J 8 Hz, 1H, Ar-H), $3.43-3.39$ (m, 2H, CH2-C=O), $2.22-2.15$ (m, 1H, Aliph-H), 1.82 - 1.78 (m, 1H, Aliph-H), 1.29 - 1.21 (m, 25H, Aliph-H), $0.93-0.84$ (m, 6H, Aliph-H ). ${ }^{13} \mathrm{C}$ NMR (100 MHz, DMSO- $\left.d_{6}\right) \delta 172.3(\mathrm{C}=\mathrm{O}), 145.4(\mathrm{C}-\mathrm{N}=\mathrm{N}), 130.5(\mathrm{C}-\mathrm{N}), 126.2$ (Ar-C), 119.8 (Ar-C), 113.9 (Ar-C), 34.7 (Aliph-C), 31.1 (Aliph-C), 28.9 (Aliph-C), 28.6 (Aliph- 
C), 28.2 (Aliph-C), 23.5 (Aliph-C), $21.9\left(\mathrm{CH}_{2}-\mathrm{CH}_{3}\right), 13.7\left(\mathrm{CH}_{3}\right)$. Anal. Calcd for $\mathrm{C}_{24} \mathrm{H}_{39} \mathrm{~N}_{3} \mathrm{O}$ : C, 74.76; H, 10.19; N, 10.90. Found: C, 74.89; H, 10.24; N, 11.12.

(S)-N-(1-(1H-1,2,3-Benzotriazol-1-yl)-3-(1H-indol-3-yl)-1-oxopropan-2-yl)-4-methyl benzenesulfonamide (3c). Brown microcrystals, yield $0.44 \mathrm{~g}(96 \%)$; mp $175-176{ }^{\circ} \mathrm{C} .{ }^{1} \mathrm{H} \mathrm{NMR}$ $\left(400 \mathrm{MHz}, \mathrm{DMSO}-d_{6}\right) \delta 10.77(\mathrm{~s}, 1 \mathrm{H}, \mathrm{NH}), 8.90\left(\mathrm{~d}, J 8.4 \mathrm{~Hz}, 1 \mathrm{H}, \mathrm{NH}-\mathrm{SO}_{2}\right), 8.23(\mathrm{~d}, J 8.0 \mathrm{~Hz}$, 1H, Ar-H), 8.03 (d, J 8.4 Hz, 1H, Ar-H), $7.77(\mathrm{t}, J 7.8 \mathrm{~Hz}, 1 \mathrm{H}, \mathrm{Ar}-\mathrm{H}), 7.61(\mathrm{t}, J 7.6 \mathrm{~Hz}, 1 \mathrm{H}$, ArH), 7.45 (d, J 7.6 Hz, 1H, Ar-H), 7.27 - 7.25 (m, 3H, Ar-H), ), 7.13 (s, 1H, CH-NH-C), 7.02 (t, J $7.4 \mathrm{~Hz}, 1 \mathrm{H}, \mathrm{Ar}-\mathrm{H}), 6.94-6.86(\mathrm{~m}, 3 \mathrm{H}, \mathrm{Ar}-\mathrm{H}), 5.55$ (q, J 8.2 Hz, 1H, CH-NH-SO $), 3.41$ (dd, $J$ 14.4, $5.6 \mathrm{~Hz}, 1 \mathrm{H}, \mathrm{CH}_{2}-\mathrm{CH}-\mathrm{NH}$ ), 3.12 (dd, J 14.2, $9.0 \mathrm{~Hz}, 1 \mathrm{H}, \mathrm{CH}_{2}-\mathrm{CH}-\mathrm{NH}$ ), 2.06 (s, 3H, $\left.\mathrm{CH}_{3}\right) .{ }^{13} \mathrm{C}$ NMR $\left(100 \mathrm{MHz}, \mathrm{DMSO}-d_{6}\right) \delta 171.2(\mathrm{C}=\mathrm{O}), 145.4(\mathrm{C}-\mathrm{N}=\mathrm{N}), 142.4\left(=\mathrm{C}-\mathrm{SO}_{2}\right), 136.9$ (Ar-C), 136.1 (Ar-C), 131.0 (Ar-C), 130.2 (Ar-C), 128.9 (Ar-C), 126.7 (Ar-C), 126.6 (Ar-C), 126.0 (Ar-C), 124.5 (Ar-C), 120.9 (Ar-C), 120.1 (Ar-C), 118.5 (Ar-C), 117.9 (Ar-C), 113.8 (ArC), 111.4 (Ar-C), 108.0 (Ar-C), $55.7\left(\mathrm{CH}-\mathrm{NH}-\mathrm{SO}_{2}\right), 28.2\left(\mathrm{CH}_{2}-\mathrm{CH}-\mathrm{NH}\right), 20.7\left(\mathrm{CH}_{3}\right)$. Anal. Calcd for $\mathrm{C}_{24} \mathrm{H}_{21} \mathrm{~N}_{5} \mathrm{O}_{3} \mathrm{~S}: \mathrm{C}, 62.73 ; \mathrm{H}, 4.61 ; \mathrm{N}, 15.24 ; \mathrm{S}, 6.98$. Found: C, 62.85; H, 4.68; N, 15.37; $\mathrm{S}, 7.02 \%$.

(1H-1,2,3-Benzotriazol-1-yl)(phenyl)methanone (3d). White microcrystals, yield $0.21 \mathrm{~g}$ (94\%); mp 111-112 ${ }^{\circ} \mathrm{C}$ (lit. $\left.{ }^{18} 110-112{ }^{\circ} \mathrm{C}\right) .{ }^{1} \mathrm{H}$ NMR (400 MHz, DMSO-d 6$) \delta 8.33-8.28$ (m, 2H, Ar-H), $8.13-8.10$ (m, 2H, Ar-H), $7.85-7.76(\mathrm{~m}, 2 \mathrm{H}, \mathrm{Ar}-\mathrm{H}), 7.68-7.63(\mathrm{~m}, 3 \mathrm{H}, \mathrm{Ar}-\mathrm{H}) .{ }^{13} \mathrm{C}$ NMR (100 MHz, DMSO-d $)) \delta 166.5(\mathrm{C}=\mathrm{O}), 145.2(\mathrm{C}-\mathrm{N}=\mathrm{N}), 133.5$ (Ar-C), 131.7 (Ar-C), 131.5 (Ar-C), 131.3 (Ar-C), 130.7 (Ar-C), 128.3 (Ar-C), 126.6 (Ar-C), 120.0 (Ar-C), 114.4 (Ar-C).

(1H-1,2,3-Benzotriazol-1-yl)(4-methoxyphenyl)methanone (3e). White microcrystals, yield $0.24 \mathrm{~g}(95 \%), \mathrm{mp} 103-104^{\circ} \mathrm{C}$ (lit. $\left.{ }^{19} 103-104{ }^{\circ} \mathrm{C}\right) .{ }^{1} \mathrm{H}$ NMR $\left(400 \mathrm{MHz}, \mathrm{DMSO}-d_{6}\right) \delta 8.30-8.27$ (m, 2H, Ar-H), 8.17 (d, J 9.2 Hz, 2H, Ar-H), 7.81 (t, J $8.2 \mathrm{~Hz}, 1 \mathrm{H}$, Ar-H), 7.64 (t, J 8.2 Hz, 1H, Ar-H), 7.19 (d, J $8.8 \mathrm{~Hz}, 2 \mathrm{H}, \mathrm{Ar}-\mathrm{H}), 3.91$ (s, 3H, $\left.\mathrm{OCH}_{3}\right)$.

(1H-1,2,3-Benzotriazol-1-yl)(3,4,5-trimethoxyphenyl)methanone (3f). White microcrystals, yield $0.3 \mathrm{~g}(96 \%), \mathrm{mp} 126-128{ }^{\circ} \mathrm{C}$ (lit. ${ }^{20} 126-128{ }^{\circ} \mathrm{C}$ ). ${ }^{1} \mathrm{H}$ NMR (400 MHz, DMSO- $\left.d_{6}\right) \delta 8.28$ (d, J $10.8 \mathrm{~Hz}, 2 \mathrm{H}, \mathrm{Ar}-\mathrm{H}), 7.83$ (t, J $7.8 \mathrm{~Hz}, 1 \mathrm{H}, \mathrm{Ar}-\mathrm{H}), 7.65$ (t, J 7.8 Hz, 1H, Ar-H), 7.47 (s, 2H, Ar-H), $3.86\left(\mathrm{~s}, 6 \mathrm{H}, m-\mathrm{OCH}_{3}\right), 3.82\left(\mathrm{~s}, 3 \mathrm{H}, p-\mathrm{OCH}_{3}\right)$.

$\boldsymbol{N}$-[2-(1H-1,2,3-Benzotriazole-1-carbonyl)phenyl]-4-methylbenzenesulfonamide (3g). White microcrystals, yield $0.37 \mathrm{~g}(94 \%), \mathrm{mp} 148-150{ }^{\circ} \mathrm{C} .{ }^{1} \mathrm{H}$ NMR $\left(400 \mathrm{MHz}, \mathrm{DMSO}-d_{6}\right) \delta 10.06$ (s, $1 \mathrm{H}, \mathrm{NHSO}_{2}$ ), 8.29 (t, J 8.2 Hz, 2H, Ar-H), 7.85 (t, J 8.2 Hz, 1H, Ar-H), 7.78 (dd, J 7.6, $1.6 \mathrm{~Hz}$, 1H, Ar-H), 7.66 (t, J 7.2 Hz, 1H,Ar-H), 7.55 - 7.50 (m, 1H,Ar-H), 7.45 (d, J 8.0 Hz, 2H, Ar-H), 7.38 (t, J 7.6 Hz, 1H, Ar-H), 7.24 (d, J 8.0 Hz, 2H, Ar-H), 7.04 (d, J 8.4 Hz, 1H, Ar-H), 2.27 (s, $\left.3 \mathrm{H}, \mathrm{CH}_{3}\right) .{ }^{13} \mathrm{C}$ NMR (100 MHz, DMSO-d 6$) \delta 165.7(\mathrm{C}=\mathrm{O}), 145.4(\mathrm{C}-\mathrm{N}=\mathrm{N}), 143.2(\mathrm{C}-\mathrm{NH})$, 136.2 (Ar-C), 135.3 (Ar-C), 132.6 (Ar-C), 131.3 (Ar-C), 131.3 (Ar-C), 130.5 (Ar-C), 129.4 (ArC), 128.6 (Ar-C), 126.6 (Ar-C), 126.4 (Ar-C), 125.5 (Ar-C), 125.2 (Ar-C), 119.9 (Ar-C), 114.3 (Ar-C), $20.8\left(\mathrm{CH}_{3}\right)$. Anal. Calcd for $\mathrm{C}_{20} \mathrm{H}_{16} \mathrm{~N}_{4} \mathrm{O}_{3} \mathrm{~S}: \mathrm{C}, 61.21 ; \mathrm{H}, 4.11 ; \mathrm{N}, 14.28 ; \mathrm{S}, 8.17$. Found: C, 61.39; H, 4.17; N, 14.45; S, 8.29\%.

(1H-1,2,3-Benzotriazol-1-yl)(3-nitrophenyl)methanone (3h). White microcrystals, yield $0.25 \mathrm{~g}$ (93\%); mp 159-162 ${ }^{\circ} \mathrm{C}$ (lit. ${ }^{9} 155-156{ }^{\circ} \mathrm{C}$ ). ${ }^{1} \mathrm{H}$ NMR (400 MHz, DMSO-d $)_{6} \delta 8.91$ (t, J $2.0 \mathrm{~Hz}$, 
1H, Ar-H), $8.60-8.57$ (m, 1H, Ar-H), $8.54-8.51$ (m, 1H, Ar-H), 8.34 (dd, J 16.0, 8.0 Hz, 2H, Ar-H), 7.95 (t, J $8.0 \mathrm{~Hz}, 1 \mathrm{H}, \mathrm{Ar}-\mathrm{H}), 7.89-7.85$ (m, 1H, Ar-H), $7.72-7.67$ (m, 1H, Ar-H). ${ }^{13} \mathrm{C}$ NMR (100 MHz, DMSO- $\left.d_{6}\right) \delta 164.7(\mathrm{C}=\mathrm{O}), 147.3\left(\mathrm{C}-\mathrm{NO}_{2}\right), 145.2(\mathrm{C}-\mathrm{N}=\mathrm{N}), 137.2(\mathrm{C}=\mathrm{CH}-$ $\mathrm{CH}=\mathrm{C}-\mathrm{NO}_{2}$ ), 133.2 (Ar-C), 131.5 (Ar-C), 131.0 (Ar-C), 130.1 (Ar-C), 127.5 (Ar-C), 126.8 (ArC), 125.9 (Ar-C), 120.1 (Ar-C), 114.4 (Ar-C).

(1H-1,2,3-Benzotriazol-1-yl)(4-nitrophenyl)methanone (3i). White microcrystals, yield 0.242 g (90\%), mp 194-196 ${ }^{\circ} \mathrm{C}$ (lit. $\left.{ }^{9} 203-205{ }^{\circ} \mathrm{C}\right) .{ }^{1} \mathrm{H}$ NMR (400 MHz, DMSO-d $\left.d_{6}\right) \delta 8.45$ (d, J $8.8 \mathrm{~Hz}$, 2H, Ar-H), 8.39 - 8.28 (m, 4H, Ar-H), 7.87 (t, J 8.2 Hz, 1H, Ar-H), 7.69 (t, J 8.4 Hz, 1H, Ar-H).

(1H-1,2,3-Benzotriazol-1-yl)(3-chlorophenyl)methanone (3j). White microcrystals, yield 0.24 g (93\%), mp 116-119 ${ }^{\circ} \mathrm{C}$ (lit. ${ }^{9} 116.5-118{ }^{\circ} \mathrm{C}$ ). ${ }^{1} \mathrm{H}$ NMR $\left(400 \mathrm{MHz}, \mathrm{DMSO}-d_{6}\right) \delta 8.30(\mathrm{t}, J 8.6 \mathrm{~Hz}$, 2H, Ar-H), 8.14 (s, 1H, CCl-CH=C-C=O), 8.06 (d, J $8.0 \mathrm{~Hz}, 1 \mathrm{H}, \mathrm{Ar}-\mathrm{H}), 7.88-7.82$ (m, 2H, Ar$\mathrm{H}), 7.70-7.63(\mathrm{~m}, 2 \mathrm{H}, \mathrm{Ar}-\mathrm{H}) .{ }^{13} \mathrm{C} \mathrm{NMR}\left(100 \mathrm{MHz}, \mathrm{DMSO}-d_{6}\right) \delta 165.2(\mathrm{C}=\mathrm{O}), 145.2(\mathrm{C}-\mathrm{N}=\mathrm{N})$, 133.5 (C-Ar), 133.1 (C-Ar), 132.9 (C-Ar), 131.6 (C-Ar), 130.8 (C-Ar), 130.76 (C-Ar), 130.3 (CAr), 129.8 (C-Ar), 126.7 (C-Ar), 120.1 (C-Ar), 114.4 (C-Ar).

(3-Aminophenyl)(1H-1,2,3-Benzotriazol-1-yl)methanone (3k). Yellow microcrystals, yield $0.22 \mathrm{~g}(92 \%)$, mp 171-172 ${ }^{\circ} \mathrm{C} .{ }^{1} \mathrm{H}$ NMR (400 MHz, DMSO- $\left.d_{6}\right) \delta 8.23$ (dd, $J 10.8,8.8 \mathrm{~Hz}, 2 \mathrm{H}$, Ar-H), 7.96 (d, J 8.4 Hz, 2H, Ar-H), 7.75 (t, J 7.4 Hz, 1H, Ar-H), 7.59 (t, J 7.4 Hz, 1H, Ar-H), $6.70(\mathrm{~d}, J 8.8 \mathrm{~Hz}, 2 \mathrm{H}, \mathrm{Ar}-\mathrm{H}), 6.48\left(\mathrm{~s}, 2 \mathrm{H}, \mathrm{NH}_{2}\right) .{ }^{13} \mathrm{C}$ NMR $\left(100 \mathrm{MHz}, \mathrm{DMSO}-d_{6}\right) \delta 165.5(\mathrm{C}=\mathrm{O})$, $155.4\left(\mathrm{C}-\mathrm{NH}_{2}\right), 145.5(\mathrm{C}-\mathrm{N}=\mathrm{N}), 135.3$ (Ar-C), 132.8 (Ar-C), 132.1 (Ar-C), 131.4 (Ar-C), 130.8 (Ar-C), 126.9 (Ar-C), 120. 2 (C-Ar), 116.9 (C-Ar), 114.8 (C-Ar), 113.6 (Ar-C), Anal. calcd for $\mathrm{C}_{13} \mathrm{H}_{10} \mathrm{~N}_{4} \mathrm{O}$ : C, 65.54; H, 4.23; N, 23.52; found: C, 65.69; H, 4.28; N, 23.74\%.

(4-Aminophenyl)(1H-1,2,3-Benzotriazol-1-yl)methanone (3l). Yellow microcrystals, yield $0.225 \mathrm{~g}(95 \%), \mathrm{mp} 178-180{ }^{\circ} \mathrm{C} .{ }^{1} \mathrm{H}$ NMR (400 MHz, DMSO-d $d_{6} \delta 8.21(\mathrm{t}, J 8.2 \mathrm{~Hz}, 2 \mathrm{H}, \mathrm{Ar}-\mathrm{H})$, $7.96(\mathrm{~d}, J 7.6 \mathrm{~Hz}, 2 \mathrm{H}, \mathrm{Ar}-\mathrm{H}$ ), 7.74 (d, J $8 \mathrm{~Hz}, 1 \mathrm{H}, \mathrm{Ar}-\mathrm{H}$ ), 7.58 (d, J $6.8 \mathrm{~Hz}, 1 \mathrm{H}, \mathrm{Ar}-\mathrm{H}), 6.71$ (d, $J 7.6 \mathrm{~Hz}, 2 \mathrm{H}, \mathrm{Ar}-\mathrm{H}), 6.51\left(\mathrm{~s}, 2 \mathrm{H}, \mathrm{NH}_{2}\right) .{ }^{13} \mathrm{C}$ NMR (100 MHz, DMSO-d $\left.d_{6}\right) \delta 164.6(\mathrm{C}=\mathrm{O}), 155.0$ $\left(\mathrm{C}-\mathrm{NH}_{2}\right), 144.9(\mathrm{C}-\mathrm{N}=\mathrm{N}), 134.6$ (Ar-C), 132.2 (Ar-C), 130.0 (Ar-C), 126.0 (Ar-C), 119.7 (Ar-C), 115.9 (Ar-C), 114.3 (Ar-C), 112.6 (Ar-C). Anal. calcd for $\mathrm{C}_{13} \mathrm{H}_{10} \mathrm{~N}_{4} \mathrm{O}: \mathrm{C}, 65.54 ; \mathrm{H}, 4.23 ; \mathrm{N}$, 23.52; found: C, 65.60; H, 4.30; N, 23.91\%.

(1H-1,2,3-Benzotriazol-1-yl)(pyridin-3-yl)methanone (3m). White microcrystals, yield $0.21 \mathrm{~g}$ (94\%), mp 101-102 ${ }^{\circ} \mathrm{C}$ (lit. $\left.{ }^{21} 101-102{ }^{\circ} \mathrm{C}\right) .{ }^{1} \mathrm{H}$ NMR (400 MHz, DMSO-d $\left.)\right) \delta 9.22$ (s, 1H, N=CH$\mathrm{C}-\mathrm{C}=\mathrm{O}), 8.90$ (d, J 6.5 Hz, 1H, Ar-H), 8.50 - 8.48 (m, 1H, Ar-H), 8.33 (dd, J 17.6 Hz, 8.4 Hz, 2H, Ar-H), $7.86(\mathrm{t}, J 7.8 \mathrm{~Hz}, 1 \mathrm{H}, \mathrm{Ar}-\mathrm{H}), 7.71-7.66(\mathrm{~m}, 2 \mathrm{H}, \mathrm{Ar}-\mathrm{H}) .{ }^{13} \mathrm{C} \mathrm{NMR}(100 \mathrm{MHz}$, DMSO- $\left.d_{6}\right) \delta 165.3(\mathrm{C}=\mathrm{O}), 153.3(\mathrm{CH}-\mathrm{N}), 151.3(\mathrm{~N}=\mathrm{CH}-\mathrm{C}-\mathrm{C}=\mathrm{O}), 145.2(\mathrm{C}-\mathrm{N}=\mathrm{N}), 138.8(\mathrm{Ar}-\mathrm{C})$, 131.4 (Ar-C), 130.9 (Ar-C), 128.0 (Ar-C), 126.8 (Ar-C), 123.3 (Ar-C), 120.1 (Ar-C), 114.3 (Ar-C).

$\mathrm{N}$-(5-(1H-1,2,3-Benzotriazole-1-carbonyl)-4-methylthiazol-2-yl)-3,4,5-trimethoxybenzamide (3n). White microcrystals, yield $0.42 \mathrm{~g}(93 \%)$, mp 107-110 ${ }^{\circ} \mathrm{C} .{ }^{1} \mathrm{H}$ NMR (400 MHz, DMSO- $d_{6}$ ) $\delta 8.29$ (d, J 8.4 Hz, 2H, Ar-H), 7.83 (t, J $6.8 \mathrm{~Hz}, 1 \mathrm{H}, \mathrm{Ar}-\mathrm{H}), 7.66$ (t, J 7.0 Hz, 1H, Ar-H), 7.47 (s, $2 \mathrm{H}, \mathrm{Ar}-\mathrm{H}), 7.14(\mathrm{~s}, 1 \mathrm{H}, \mathrm{NH}), 3.86\left(\mathrm{~s}, 6 \mathrm{H}, m-\left(\mathrm{OCH}_{3}\right)\right), 3.82\left(\mathrm{~s}, 3 \mathrm{H}, p-\left(\mathrm{OCH}_{3}\right)\right) 1.17\left(\mathrm{~s}, 3 \mathrm{H}, \mathrm{CH}_{3}\right)$ ${ }^{13} \mathrm{C}$ NMR $\left(100 \mathrm{MHz}, \mathrm{DMSO}-d_{6}\right) \delta 165.80(\mathrm{C}=\mathrm{O}), 152.42(\mathrm{C}=\mathrm{O}), 145.2\left(\mathrm{~N}-\mathrm{C}-\mathrm{CH}_{3}\right), 142.3(\mathrm{C}-$ 
$\mathrm{OCH}_{3}$ ), 131.9 (Ar-C), 130.7 (Ar-C), 128.2 (Ar-C),127.4 (Ar-C), 126.6 (Ar-C), 126.2 (Ar-C), 125.7 (Ar-C), 120.0 (Ar-C), 114.3 (Ar-C), 109.4 (Ar-C), $60.3\left(p-\mathrm{OCH}_{3}\right), 56.2\left(m-\mathrm{OCH}_{3}\right), 14.1$ $\left(\mathrm{CH}_{3}\right)$. Anal. Calcd for $\mathrm{C}_{21} \mathrm{H}_{19} \mathrm{~N}_{5} \mathrm{O}_{5} \mathrm{~S}: \mathrm{C}, 55.62 ; \mathrm{H}, 4.22 ; \mathrm{N}, 15.44 ; \mathrm{S}, 7.07$. Found: C, 55.78; H, $4.30 ; \mathrm{N}, 15.61 ; \mathrm{S}, 7.15 \%$.

Synthesis of 8-(1H-1,2,3-benzotriazol-1-yl)-8-oxooctanoic acid (5). A mixture of $p$ toluenesulfonyl chloride $(0.38 \mathrm{~g}, 2 \mathrm{mmol})$ and DMAP $(0.032 \mathrm{~g}, 0.26 \mathrm{mmol})$ was stirred in $\mathrm{CH}_{2} \mathrm{Cl}_{2}(10 \mathrm{~mL})$ for 10 minutes. Suberic acid $(0.348 \mathrm{~g}, 2 \mathrm{mmol})$ was dissolved in $\mathrm{CH}_{2} \mathrm{Cl}_{2}(10$ $\mathrm{mL})$ containing TEA $(0.7 \mathrm{~mL}, 5 \mathrm{mmol})$ and the resulting solution was added to the reaction mixture. After 20 minutes, benzotriazole $(0.286 \mathrm{~g}, 2.4 \mathrm{mmol})$ was added and the reaction was allowed to stir for additional $1.5 \mathrm{~h}$ at $25{ }^{\circ} \mathrm{C}$. The reaction was diluted with $\mathrm{CH}_{2} \mathrm{Cl}_{2}(50 \mathrm{~mL})$ and the organic layer was washed with $6 \mathrm{~N} \mathrm{HCl}(3 \times 10 \mathrm{~mL})$, water $(2 \times 10 \mathrm{~mL})$ and brine $(1 \times 10$ $\mathrm{mL})$. The organic layer was dried over anhydrous sodium sulfate and hexane $(20 \mathrm{~mL})$ was added. The solid separated was filtered and dried under vacuum to give compound $5(0.522 \mathrm{~g}, 95 \%)$.

8-(1H-1,2,3-Benzotriazol-1-yl)-8-oxooctanoic acid (5). White microcrystals, mp $118-120{ }^{\circ} \mathrm{C}$. ${ }^{1} \mathrm{H}$ NMR (400 MHz, DMSO-d $\left.)\right) \delta 11.94$ (s, 1H, COOH), 8.23 (d, J 9.3 Hz, 2H, Ar-H), 7.79$7.74(\mathrm{~m}, 1 \mathrm{H}, \mathrm{Ar}-\mathrm{H}), 7.62-7.58(\mathrm{~m}, 1 \mathrm{H}, \mathrm{Ar}-\mathrm{H}), 3.47-3.38\left(\mathrm{~m}, 2 \mathrm{H},-\mathrm{CH}_{2}-\mathrm{CO}-\mathrm{N}\right), 2.21$ (t, J 7.4 $\left.\mathrm{Hz}, 2 \mathrm{H}, \mathrm{CH}_{2}-\mathrm{COOH}\right), 1.84-1.75$ (m, 2H, Aliph-H), 1.57 - 1.49 (m , 2H, Aliph-H), $1.45-1.33$ (m, 4H, Aliph-H). ${ }^{13} \mathrm{C}$ NMR (100 MHz, DMSO-d $) \delta 174.4(\mathrm{COOH}), 172.3(\mathrm{~N}-\mathrm{C}=\mathrm{O}), 145.4(\mathrm{C}-$ $\mathrm{N}=\mathrm{N}), 130.6$ (C-N), 130.5 (C-Ar), 126.2 (C-Ar), 119.9 (C-Ar), 113.9 (C-Ar), $34.7\left(\mathrm{CH}_{2} \mathrm{COOH}\right)$, 33.5 (CH2-CO-N), 28.2 (C-Aliph), 28.0 (C-Aliph), 24.3, (C-Aliph) 23.4 (C-Aliph). Anal. Calcd for $\mathrm{C}_{14} \mathrm{H}_{17} \mathrm{~N}_{3} \mathrm{O}_{3}$ : C, 61.08; H, 6.22; N, 15.26. Found: C, 61.29; H, 6.34; N, 15.43\%.

Synthesis of suberanilic acid (6). To a solution of $5(0.413 \mathrm{~g}$., $1.5 \mathrm{mmol})$ in $\mathrm{CH}_{2} \mathrm{Cl}_{2}(20 \mathrm{~mL})$ was added aniline $(0.27 \mathrm{~mL}, 3 \mathrm{mmol})$ and the mixture as stirred for $1 \mathrm{~h}$ at $25{ }^{\circ} \mathrm{C}$. The reaction was diluted with $\mathrm{CH}_{2} \mathrm{Cl}_{2}(40 \mathrm{~mL})$ then, washed with $6 \mathrm{~N} \mathrm{HCl}(3 \times 10 \mathrm{~mL})$, water $(2 \times 10 \mathrm{~mL})$ and brine $(1 \times 10 \mathrm{~mL})$. The organic layer was dried over anhydrous sodium sulfate and the filtrate was evaporated to give compound $6(0.36 \mathrm{~g}, 96 \%)$.

8-Oxo-8-(phenylamino)octanoic acid (6). White powder, mp $114-116{ }^{\circ} \mathrm{C}$ (lit. ${ }^{15} 126-128{ }^{\circ} \mathrm{C}$ ). ${ }^{1} \mathrm{H}$ NMR (400 MHz, DMSO-d $)_{6} \delta 11.94(\mathrm{~s}, 1 \mathrm{H}, \mathrm{COOH}), 9.81(\mathrm{~s}, 1 \mathrm{H}, \mathrm{NH}), 7.58(\mathrm{~d}, J 7.2 \mathrm{~Hz}, 2 \mathrm{H}$, Ar-H), 7.32 - 7.22 (m, 2H, Ar-H), $7.06-6.96$ (m, 1H, Ar-H), 2.29 (t, J 7.6 Hz, 2H, CH $2-C O-$ $\mathrm{NH}-$ ), 2.19 (t, J 7.4 Hz, 2H, $\mathrm{CH}_{2} \mathrm{COOH}$ ), $1.66-1.57$ (m, 2H, $\mathrm{CH}_{2} \mathrm{CH}_{2} \mathrm{CONH}-$ ), $1.53-1.47$ (m, $2 \mathrm{H}, \mathrm{CH}_{2} \mathrm{CH}_{2} \mathrm{COOH}$ ), $1.33-1.28$ (m, 4H, Aliph-H). ${ }^{13} \mathrm{C}$ NMR (100 MHz, DMSO-d $) \delta 174.35$ (CO-NH), 171.12 (COOH), 139.28 (C-Ar), 128.53 (C-Ar), 122.81 (C-Ar), 118.98 (C-Ar), 36.32 $\left(\mathrm{CH}_{2} \mathrm{CONH}\right), 33.56\left(\mathrm{CH}_{2} \mathrm{COOH}\right), 28.43$ (C-Aliph), 28.26 (C-Aliph), 24.96 (C-Aliph), 24.32 (CAliph).

Synthesis of 8-(1H-1,2,3-benzotriazol-1-yl)-8-oxo- $N$-phenyloctanamide (7). A mixture of $p$ toluenesulfonyl chloride $(0.19 \mathrm{~g}, 1 \mathrm{mmol})$ and DMAP $(0.016 \mathrm{~g}, 0.13 \mathrm{mmol})$ was stirred in $\mathrm{CH}_{2} \mathrm{Cl}_{2}(5 \mathrm{~mL})$ for 10 minutes. Compound 6 (0.249 g., $\left.1 \mathrm{mmol}\right)$ was dissolved in $\mathrm{CH}_{2} \mathrm{Cl}_{2}(5 \mathrm{~mL})$ containing TEA $(0.21 \mathrm{~mL}, 1.5 \mathrm{mmol})$ and the resulting solution was added to the reaction mixture. After 20 minutes, benzotriazole $(0.143 \mathrm{~g}, 1.2 \mathrm{mmol})$ was added and the reaction was allowed to stir for additional $1.5 \mathrm{~h}$ at $25{ }^{\circ} \mathrm{C}$. The reaction was diluted with $\mathrm{CH}_{2} \mathrm{Cl}_{2}(50 \mathrm{~mL})$ and 
the organic layer was washed with saturated $\mathrm{Na}_{2} \mathrm{CO}_{3}(3 \times 10 \mathrm{~mL})$, water $(2 \times 10 \mathrm{~mL})$ and brine $(1 \times 10 \mathrm{~mL})$. The organic layer was dried over anhydrous sodium sulfate and hexane $(20 \mathrm{~mL})$ was added. The solid separated was filtered and dried under vacuum to give compound 7 (0.34 $\mathrm{g}$, $97.1 \%)$.

8-(1H-1,2,3-Benzotriazol-1-yl)-8-oxo-N-phenyloctanamide (7). Grayish-white powder: mp 77$79{ }^{\circ} \mathrm{C} .{ }^{1} \mathrm{H}$ NMR $\left(400 \mathrm{MHz}, \mathrm{DMSO}-d_{6}\right) \delta 9.85(\mathrm{~s}, 1 \mathrm{H}, \mathrm{NH}), 8.23(\mathrm{~d}, J 8.8 \mathrm{~Hz}, 2 \mathrm{H}, \mathrm{Ar}-\mathrm{H}), 7.76(\mathrm{t}$, $J 7.8 \mathrm{~Hz}, 1 \mathrm{H}, \mathrm{Ar}-\mathrm{H}), 7.59$ (t, J 8.0 Hz, 3H, Ar-H), 7.27 (t, J 7.8 Hz, 2H, Ar-H), 7.00 (t, J 7.4 Hz, 1H, Ar-H), 3.41 (t, J 7.4 Hz, 2H, $\mathrm{CH}_{2} \mathrm{CON}-\mathrm{N}$ ), 2.30 (q, J 7.7 Hz, 2H, $\mathrm{CH}_{2} \mathrm{CONH}$ ), $1.84-1.76$ (m, 2H, Aliph-h), $1.66-1.60$ (m,2H, Aliph-H), $1.50-1.32$ (m, 4H, Aliph-H). ${ }^{13} \mathrm{C}$ NMR (100 MHz, DMSO- $\left.d_{6}\right) \delta 172.4(\mathrm{CONH}), 171.2(\mathrm{CON}-\mathrm{N}), 145.4(\mathrm{C}-\mathrm{N}=\mathrm{N}), 139.3(\mathrm{C}-\mathrm{Ar}), 130.6(\mathrm{C}-$ Ar), 130.6 (C-Ar), 128.6 (C-Ar), 126.3 (C-Ar), 122.9 (C-Ar), 119.9 (C-Ar), 119.0 (C-Ar), 114.0 (C-Ar), $36.3\left(\mathrm{CH}_{2} \mathrm{CONH}\right), 34.8\left(\mathrm{CH}_{2} \mathrm{CON}-\mathrm{N}\right), 28.4$ (C-Aliph), 28.2 (C-Aliph), 24.9 (C-Aliph), 23.43 (C-Aliph). Anal. Calcd for $\mathrm{C}_{20} \mathrm{H}_{22} \mathrm{~N}_{4} \mathrm{O}_{2}$ : C, 68.55; H, 6.33; N, 15.99. Found: C, 68.69; H, $6.41 ; \mathrm{N}, 16.14$.

Synthesis of Vorinostate $(8)$. To a solution of $7(0.175 \mathrm{~g}, 0.5 \mathrm{mmol})$ in $\mathrm{CH}_{2} \mathrm{Cl}_{2}(10 \mathrm{~mL})$ was added hydroxylamine $\mathrm{HCl}(0.069 \mathrm{~g}, 1 \mathrm{mmol})$ and TEA $(0.14 \mathrm{~mL}, 1 \mathrm{mmol})$. The mixture was stirred at $25{ }^{\circ} \mathrm{C}$ for $1 \mathrm{~h}$. The reaction was diluted with $\mathrm{CH}_{2} \mathrm{Cl}_{2}(40 \mathrm{~mL})$ then, washed with $6 \mathrm{~N} \mathrm{HCl}$ $(3 \times 10 \mathrm{~mL})$, water $(2 \times 10 \mathrm{~mL})$ and brine $(1 \times 10 \mathrm{~mL})$. The organic layer was dried over anhydrous sodium sulfate and the filtrate was evaporated to afford compound $\mathbf{8}$ (yield $0.125 \mathrm{~g}$, $95 \%)$.

$\boldsymbol{N}^{\mathbf{1}}$-hydroxy- $\boldsymbol{N}^{\mathbf{8}}$-phenyloctanediamide (8). Pale orange microcrystals, mp 161-162 ${ }^{\circ} \mathrm{C}$ (lit. ${ }^{15}$ 159-161 ${ }^{\circ} \mathrm{C}$ ). ${ }^{1} \mathrm{H}$ NMR (400 MHz, DMSO- $\left.d_{6}\right) \delta 9.84$ (s, $\left.1 \mathrm{H}, \mathrm{NHCOCH}_{2}\right), 8.50(\mathrm{~s}, 1 \mathrm{H}, \mathrm{NHOH})$, $8.20(\mathrm{~s}, 1 \mathrm{H}, \mathrm{OH}), 7.58$ (d, J 7.6 Hz, 2H, Ar-H), 7.27 (t, J 7.8 Hz, 2H, Ar-H), 7.01 (t, J 7.4 Hz, 1H, Ar-H), 3.03 (q, J $6.7 \mathrm{~Hz}, 1 \mathrm{H}$, Aliph-H), 2.32 - 2.25 (m, 3H, Aliph-H), $1.65-1.52$ (m, 3H, Aliph-H), $1.44-1.39$ (m, 1H, Aliph-H), 1.35 - 1.25 (m, 4H, Aliph-H). ${ }^{13} \mathrm{C}$ NMR (100 MHz, DMSO-d $\left.d_{6}\right) \delta 171.2$ (CONH), 161.4 (CO-NH-OH) 139.3 (C-Ar), 128.5 (C-Ar), 122.8 (C-Ar), 119.0 (C-Ar), 36.4 ( $\left.\mathrm{CH}_{2}-\mathrm{CONH}\right), 29.8$ ( $\left.\mathrm{CH}_{2} \mathrm{CONHOH}\right), 28.4$ (C-Aliph), 26.1 (C-Aliph), 25.0 (C-Aliph), 24.9 (C-Aliph).

\section{Supplementary material available}

Detailed NMR spectra are presented in the Supplementary Data file.

\section{References}

1. Katritzky, A. R.; Drewniak, M. J. Chem. Soc. Perkin Trans. 1. 1988, 2339. http://dx.doi.org/10.1039/P19880002339.

2. Katritzky, A. R.; Suzuki, K.; Wang, Z. Synlett 2005, 11, 1656. http://dx.doi.org/10.1055/s-2005-871551. 
3. Abo-Dya, N. E.; Biswas, S.; Basak, A.; Avan, I.; Alamry, K. A.; Katritzky, A. R. J. Org. Chem 2013, 78, 3541. http://dx.doi.org/10.1021/jo302251e.

4. Biswas, S.; Abo-Dya, N. E.; Oliferenko, A.; Khiabani, A.; Steel, P. J.; Alamry, K. A.; Katritzky, A. R. J. Org. Chem. 2013, 78, 8502. http://dx.doi.org/10.1021/jo401234g.

5. Panda, S. S.; Hall, C. D.; Scriven, E.; Katritzky, A. R. Aldrichim. Acta 2013, 46, 43. http://dx.doi.org/10.1002/chin.201441274.

6. Beagle, L. K.; Hansen, F. K.; Monbaliu, J.-C. M.; Des Rosiers, M. P.; Phillips, A. M.; Stevens, C. V.; Katritzky, A. R. Synlett 2012, 23, 2337. http://dx.doi.org/10.1055/s-0031-1290446.

7. El-Khatib, M.; Elagawany, M.; Caliskan, E.; Davis, E. F.; Faidallah, H. M.; El-Feky, S. A.; Katritzky, A. R. Chem. Commun. 2013, 49, 2631. http://dx.doi.org/10.1039/c3cc39291k.

8. Katritzky, A. R.; Tala, S. R.; Abo-Dya, N. E.; Ibrahim, T. S.; El-Feky, S. A.; Gyanda, K.; Pandya, K. M. J. Org. Chem. 2011, 76, 85. http://dx.doi.org/10.1021/jo1015757.

9. Duangkamol, C.; Wangngae, S.; Pattarawarapan, M.; Phakhodee, W. Eur. J. Org. Chem. 2014, 7109. http://dx.doi.org/10.1002/ejoc.201403076.

10. Wetosot, S.; Duangkamol, C.; Pattarawarapan, M.; Phakhodee, W. Monatsh. Chem. 2015, $146,959$. http://dx.doi.org/10.1007/s00706-014-1408-1.

11. Theodoropoulos, D.; Gazopoulos, J. J. Org. Chem. 1962, 27, 2091. http://dx.doi.org/10.1021/jo01053a045

12. Khalafi-Nezhad, A.; Parhami, A.; Zare, A.; Moosavi Zare, A. R. J. Iran. Chem. Soc. 2008, 5, 413. http://dx.doi.org/10.1007/BF03245996.

13. Berry, D. J.; DiGiovanna, C. V; Metrick, S. S.; Murugan, R. Arkivoc 2001, 2, 201.

14. Breslow, R.; Marks, P. A.; Rifkind, R. A.; Jursic, B. PTC Int. Appl. WO 93/07148 April 15, 1993.

15. Mai, A.; Esposito, M.; Sbardella, G.; Massa, S. Org. Prep. Proced. Int. 2001, 33, 391. http://dx.doi.org/10.1080/00304940109356608

16. Day, J. A.; Cohen, S. M. J. Med. Chem. 2013, 56, 7997. http://dx.doi.org/10.1021/jm401053m.

17. Reboud-R., M.; Ghelis, C. Eur. J. Biochem. 1976, 65, 25. http://dx.doi.org/10.1111/j.1432-1033.1976.tb10385.x

18. Katritzky, A. R.; Vakulenko, A.; Jain, R. Arkivoc 2003, xiv, 131.

19. Katritzky, A. R.; Cai, C.; Singh, S. K. J. Org. Chem. 2006, 71, 3375. http://dx.doi.org/10.1021/jo052443x 
20. Fu, J.; Yang, Y.; Zhang, X.-W.; Mao, W.-J.; Zhang, Z.-M.; Zhu, H.-L. Bioorg. Med. Chem. 2010, 18, 8457.

http://dx.doi.org/10.1016/j.bmc.2010.10.049.

21. El-Nachef, C.; Bajaj, K.; Koblick, J.; Katritzky, A. R. Eur. J. Org. Chem. 2012, 4412. http://dx.doi.org/10.1002/ejoc.201200323. 